\title{
Heck Couplings at Room Temperature in Nanometer Aqueous Micelles
}

\author{
Bruce H. Lipshutz* and Benjamin R. Taft \\ Department of Chemistry \& Biochemistry \\ University of California \\ Santa Barbara, CA 93106
}

\section{Supporting Information}

Table of Contents:

- General information: S2

- 15 wt.\% PTS $/ \mathrm{H}_{2} \mathrm{O}$ preparation: S2

- General Heck coupling procedure: S2

- Notes: S2

- Compound characterization data: S3-S6

- ${ }^{1} \mathrm{H}$ and ${ }^{13} \mathrm{C}$ NMR spectra: S7-S16 
General. Reactions were performed in non-oven-dried glassware under an Argon atmosphere containing a Teflon coated stir bar and septum. Water (HPLC grade) was purchased from ACROS and degassed prior to use. All commercially available reagents were used without further purification. (dtbpf) $\mathrm{PdCl}_{2}$ (7) was generously supplied by Johnson Matthey, PTS and PSS where supplied by Zymes, and TPGS was supplied by Eastman. SDS and PEG400 were purchased from Aldrich. Triton X-100 was purchased from ACROS. TLC analyses were performed on commercial Kieselgel 60 F254 silica gel plates. GC analyses were performed on an HP-5 capillary column $(0.25 \mu$ x $30 \mathrm{~m}$; cross-linked 5\% PHME siloxane) and a time program beginning with $5 \mathrm{~min}$ at $50{ }^{\circ} \mathrm{C}$ followed by $20{ }^{\circ} \mathrm{C} / \mathrm{min}$ ramp to $280{ }^{\circ} \mathrm{C}$, then $20 \mathrm{~min}$ at this temp. Column chromatography was performed using Davisil Grade 633 Type $60 \AA ̊$ silica gel. NMR spectra were obtained on a Varian Inova system using $\mathrm{CDCl}_{3}$ as solvent, with proton and carbon resonances at $400 \mathrm{MHz}$ and $100 \mathrm{MHz}$, respectively. Mass spectral data were acquired on a VF Autospec or an analytical VG-70-250 HF instrument. Dynamic light scattering (DLS) was performed on a Brookhaven Instruments particle size analyzer equipped with an Avalanche photodiode detector and a MG Vertically polarized $35 \mathrm{~mW}$ He-Ne $633 \mathrm{~nm}$ laser. Cryo-TEM images were obtained using a JEOL JEM\#8209;1210, 120kv, (tungsten filament) instrument.

Preparation of 15 wt.\% PTS aqueous solution: PTS $(3.0 \mathrm{~g}, \sim 3.0 \mathrm{~mL})$ was added to a $60 \mathrm{~mL}$ serum bottle along with a "large" Teflon coated stir bar. The bottle was purged with argon before addition of degassed water $(17.0 \mathrm{~mL})$ and sealed under a positive flow of argon. The mixture was stirred vigorously overnight at room temperature to give a clear, homogeneous, "soapy" solution. The solution was stored under argon on the bench-top for weeks without noticeable decomposition.

General Procedure for Heck Coupling: (dtbpf) $\mathrm{PdCl}_{2}(7)(6.5 \mathrm{mg}, 0.01 \mathrm{mmol})$ and aryl iodide $(0.50 \mathrm{mmol})$ were added under argon to a $5.0 \mathrm{~mL}$ microwave vial equipped with a large stir bar and Teflon lined septum. PTS solution ( $1.0 \mathrm{~mL}, 15 \mathrm{wt} . \%)$, triethylamine ( $208 \mu \mathrm{L}, 1.50 \mathrm{mmol})$, and acrylate/styrene $(1.0 \mathrm{mmol})$ were added by syringe. The heterogeneous mixture was stirred vigorously at $\mathrm{rt}$, becoming pseudo-homogeneous after 20-40 min. Reaction progress was monitored by TLC (5-10\% EtOAc/hexane) and/or GC. Upon consumption of aryl iodide (2-24 h), the dark brown mixture was diluted with EtOAc $(\sim 1.5 \mathrm{~mL})$ and filtered through a pad of silica gel using EtOAc as the eluent. The volatiles were removed on a rotary evaporator and the crude product was purified by silica gel chromatography or crystallization from warm EtOH. (Alternatively, the crude reaction mixture can be partitioned between hexane/EtOAc (3/1) and $\mathrm{H}_{2} \mathrm{O} /$ brine (3/1) and washed to remove the catalyst and surfactant).

\section{Notes:}

$>$ Reactions were conducted in $5.0 \mathrm{~mL}$ microwave tubes with large Teflon coated stir bars and Teflon lined septum under $1.0 \mathrm{~atm}$ of Ar.

> Oversize stir bars were used, without which solutions can be viscous and difficult to mix.

$>$ Solutions generally become a dark brown as reaction progresses, and oftentimes the product will precipitate out of solution.

$>$ Generally, cinnamate products were purified via silica gel chromatography, while stilbene derivatives were purified via crystallization from warm EtOH. 
Compound Characterization Data. Compounds not listed are known compounds; spectral data correspond to that in the literature. ${ }^{[1]}$

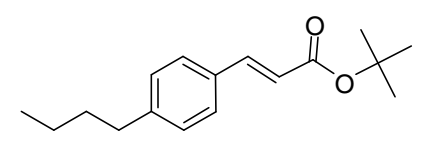

(E)-tert-Butyl 3-(4-butylphenyl)acrylate. Following the general procedure using 4-n-butyliodobenzene (130 mg, $0.5 \mathrm{mmol})$ and $t$-butyl acrylate $(145 \mu \mathrm{L}, 1.0 \mathrm{mmol})$, the reaction was stirred for $5 \mathrm{~h}$ at $\mathrm{rt}$. Following the standard workup, chromatography of the crude material on silica gel (1:20, EtOAc/hexanes) gave $101 \mathrm{mg}$ (77\%) of a tan waxy semi-solid. ${ }^{1} \mathrm{H}$ NMR $\left(400 \mathrm{MHz}, \mathrm{CDCl}_{3}\right) \delta 7.58(\mathrm{~d}, J=15.8 \mathrm{~Hz}, 1 \mathrm{H}), 7.42(\mathrm{~d}, J=8.1 \mathrm{~Hz}, 2 \mathrm{H}), 7.19(\mathrm{~d}, J=8.1$ $\mathrm{Hz}, 2 \mathrm{H}), 6.34(\mathrm{~d}, J=15.8 \mathrm{~Hz}, 1 \mathrm{H}), 2.62(\mathrm{t}, J=7.8 \mathrm{~Hz}, 2 \mathrm{H}), 1.60(\mathrm{~m}, 2 \mathrm{H}), 1.54(\mathrm{~s}, 9 \mathrm{H}), 1.37(\mathrm{~m}, 2 \mathrm{H}), 0.94(\mathrm{t}, J=$ $7.6 \mathrm{~Hz}, 3 \mathrm{H}) .{ }^{13} \mathrm{C} \mathrm{NMR}\left(100 \mathrm{MHz}, \mathrm{CDCl}_{3}\right) \delta 166.7,145.4,143.7,132.2,129.0,128.1,119.2,80.4,35.7,33.6,28.4$, 22.5, 14.1. HREIMS calcd for $\mathrm{C}_{17} \mathrm{H}_{24} \mathrm{O}_{2}\left[\mathrm{M}^{+}\right]=260.1776$, found 260.1767 .



(E)-tert-Butyl 5-(3-cholesteryloxy-3-oxoprop-1-enyl)-indole-1-carboxylate (8). Following the general procedure using (dtbpf) $\mathrm{PdCl}_{2}(3.5 \mathrm{mg}, 0.005 \mathrm{mmol}), t$-butyl 5-iodoindole-1-carboxylate (142 $\left.\mathrm{mg}, 0.5 \mathrm{mmol}\right)$, and cholesteryl acrylate $(111 \mathrm{mg}, 0.25 \mathrm{mmol})$, the reaction was stirred for $8 \mathrm{~h}$ at $\mathrm{rt}$. Following the standard workup, chromatography of the crude material on silica gel (1:10, EtOAc/hexanes) gave $138 \mathrm{mg}$ (84\%) of a colorless solid, $\mathrm{mp}=172-174{ }^{\circ} \mathrm{C}$ (decomposition). [Additionally $10 \mathrm{mg}$ of the (Z)-isomer was obtained (6\%).] ${ }^{1} \mathrm{H} \mathrm{NMR} \mathrm{(400} \mathrm{MHz,}$ $\left.\mathrm{CDCl}_{3}\right) \delta 8.14(\mathrm{~d}, J=8.4 \mathrm{~Hz}, 1 \mathrm{H}), 7.79(\mathrm{~d}, J=16.0 \mathrm{~Hz}, 1 \mathrm{H}), 7.72(\mathrm{~s}, 1 \mathrm{H}), 7.61(\mathrm{~d}, J=3.6 \mathrm{~Hz}, 1 \mathrm{H}), 7.52(\mathrm{dd}, J=7.7$, $1.5 \mathrm{~Hz}, 1 \mathrm{H}), 6.59(\mathrm{~d}, J=3.6 \mathrm{~Hz}, 1 \mathrm{H}), 6.44(\mathrm{~d}, J=16.0 \mathrm{~Hz}, 1 \mathrm{H}), 5.42(\mathrm{~d}, J=4.7 \mathrm{~Hz}, 1 \mathrm{H}), 4.77(\mathrm{~m}, 1 \mathrm{H}), 2.42(\mathrm{~d}, J=$ $7.2 \mathrm{~Hz}, 2 \mathrm{H}), 2.05-1.80(\mathrm{~m}, 6 \mathrm{H}), 1.69$ (s, 9H), 1.78-0.96 (m, 26H), 0.94 (d, $J=6.1 \mathrm{~Hz}, 2 \mathrm{H}), 0.88$ (dd, $J=6.6,1.5 \mathrm{~Hz}$, $4 \mathrm{H}), 0.70$ (s, 3H). ${ }^{13} \mathrm{C} \mathrm{NMR}\left(100 \mathrm{MHz}, \mathrm{CDCl}_{3}\right) \delta 167.2,149.9,145.6,140.2,131.4,129.7,127.4,124.4,123.2$, 122.0, 117.7, 116.0, 107.9, 94.9, 84.6, 74.4, 57.2, 56.6, 50.5, 42.8, 40.2, 40.0, 38.8, 37.5, 37.1, 36.7, 36.3, 32.4, 32.3, $28.8,28.7,28.5,28.4,24.8,24.3,23.4,23.1,21.5,19.9,19.2,12.4$. HREIMS calcd for $\mathrm{C}_{43} \mathrm{H}_{61} \mathrm{NO}_{4} \mathrm{Na}[\mathrm{M}+\mathrm{Na}]=$ 678.4498 , found 678.4521 .

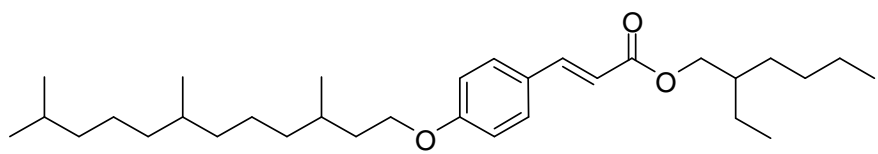

(E)-2-Ethylhexyl 3-(4-(3,7,11-trimethyldodecyloxy)phenyl)acrylate. Following the general procedure using 1iodo-4-(3,7,11-trimethyldodecyloxy)benzene ( $215 \mathrm{mg}, 0.5 \mathrm{mmol})$ and 2-ethylhexyl acrylate (208 $\mu \mathrm{L}, 1.0 \mathrm{mmol})$, the reaction was stirred for $8 \mathrm{~h}$ at $50{ }^{\circ} \mathrm{C}$. Following the standard workup, chromatography of the crude material on silica 
gel (1:20, EtOAc/hexanes) gave $229 \mathrm{mg}(94 \%)$ of a colorless oil. ${ }^{1} \mathrm{H}$ NMR $\left(400 \mathrm{MHz}, \mathrm{CDCl}_{3}\right) \delta 7.58(\mathrm{~d}, J=15.8$ $\mathrm{Hz}, 1 \mathrm{H}), 7.61(\mathrm{~d}, J=16.0 \mathrm{~Hz}, 1 \mathrm{H}), 7.46(\mathrm{~d}, J=8.0 \mathrm{~Hz}, 2 \mathrm{H}), 6.88(\mathrm{~d}, J=8.0 \mathrm{~Hz}, 2 \mathrm{H}), 6.30(\mathrm{~d}, J=16.0 \mathrm{~Hz}, 1 \mathrm{H}), 4.09$ $(\mathrm{m}, 2 \mathrm{H}), 4.01(\mathrm{~m}, 2 \mathrm{H}), 1.83(\mathrm{~m}, 1 \mathrm{H}), 1.70-1.01(\mathrm{~m}, 27 \mathrm{H}), 0.94-0.82(\mathrm{~m}, 18 \mathrm{H}) .{ }^{13} \mathrm{C} \mathrm{NMR}\left(100 \mathrm{MHz}, \mathrm{CDCl}_{3}\right) \delta$ 167.5, 160.9, 144.2, 129.6, 126.9, 115.5, 114.7, 66.7, 66.4, 39.3, 38.8, 37.3, 37.2, 36.1, 36.0, 32.7, 30.4, 29.7, 28.9, 27.9, 24.3, 23.8, 23.0, 22.7, 22.6, 19.7, 19.6, 19.5, 14.1, 11.0. HREIMS calcd for $\mathrm{C}_{32} \mathrm{H}_{54} \mathrm{O}_{3}\left[\mathrm{M}^{+}\right]=486.4073$, found 486.4064 .

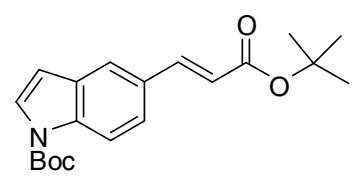

(E)-tert-Butyl 5-(3-tert-butoxy-3-oxoprop-1-enyl)-indole-1-carboxylate. Following the general procedure using $t$ butyl 5-iodoindole-1-carboxylate $(142 \mathrm{mg}, 0.5 \mathrm{mmol})$, and $t$-butyl acrylate $(145 \mu \mathrm{L}, 1.0 \mathrm{mmol})$, the reaction was stirred for $6 \mathrm{~h}$ at $\mathrm{rt}$. Following the standard workup, chromatography of the crude material on silica gel (1:10, EtOAc/hexanes) gave $164 \mathrm{mg}(96 \%)$ of a light yellow oil. $\left.{ }^{1} \mathrm{H} \mathrm{NMR} \mathrm{(400} \mathrm{MHz,} \mathrm{CDCl}_{3}\right) \delta 8.13(\mathrm{~d}, J=8.5 \mathrm{~Hz}, 1 \mathrm{H})$, $7.70(\mathrm{~m}, 2 \mathrm{H}), 7.61(\mathrm{~d}, J=3.5 \mathrm{~Hz}, 1 \mathrm{H}), 7.51(\mathrm{dd}, J=8.7,2.0 \mathrm{~Hz}, 1 \mathrm{H}), 6.59(\mathrm{~d}, J=3.8 \mathrm{~Hz}, 1 \mathrm{H}), 6.40(\mathrm{~d}, J=16.0 \mathrm{~Hz}$, $1 \mathrm{H}), 1.68$ (s, 9H), 1.56 (s, 9H). ${ }^{13} \mathrm{C} \mathrm{NMR}\left(100 \mathrm{MHz}, \mathrm{CDCl}_{3}\right) \delta 166.8,149.6,144.4,136.3,131.1,129.5,127.0$, 124.0, 121.5, 118.9, 115.6, 107.6, 84.3, 80.5, 28.5, 28.4. HREIMS calcd for $\mathrm{C}_{20} \mathrm{H}_{25} \mathrm{NO}_{4}[\mathrm{M}+\mathrm{Na}]=366.1681$, found 366.1667.

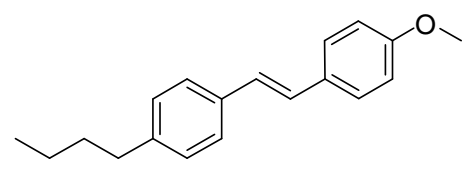

(E)-1-Butyl-4-(4-methoxystyryl)benzene. Following the general procedure using 4- $n$-butyliodobenzene (130 mg, $0.5 \mathrm{mmol})$ and 4-methoxystyrene $(133 \mu \mathrm{L}, 1.0 \mathrm{mmol})$, the reaction was stirred for $1.5 \mathrm{~h}$ at rt. Following the standard workup, recrystallization of the crude product from warm EtOH gave $115 \mathrm{mg}(86 \%$,) of a tan solid, $\mathrm{mp}=116$ $118{ }^{\circ} \mathrm{C}$. [Additionally $12 \mathrm{mg}(Z)$-isomer was isolated (10\%).] ${ }^{1} \mathrm{H} \mathrm{NMR}\left(400 \mathrm{MHz}, \mathrm{CDCl}_{3}\right) \delta 7.46(\mathrm{~d}, J=8.6 \mathrm{~Hz}$, 2H), $7.43(\mathrm{~d}, J=8.6 \mathrm{~Hz}, 2 \mathrm{H}), 7.18(\mathrm{~d}, J=8.2 \mathrm{~Hz}, 2 \mathrm{H}), 7.05(\mathrm{~d}, J=16.3 \mathrm{~Hz}, 1 \mathrm{H}), 6.97(\mathrm{~d}, J=16.3 \mathrm{~Hz}, 1 \mathrm{H}), 6.91(\mathrm{~d}$, $J=8.6 \mathrm{~Hz}, 2 \mathrm{H}), 3.84(\mathrm{~s}, 3 \mathrm{H}), 2.62(\mathrm{t}, J=7.4 \mathrm{~Hz}, 2 \mathrm{H}), 1.61(\mathrm{~m}, 2 \mathrm{H}), 1.39(\mathrm{~m}, 2 \mathrm{H}), 0.95(\mathrm{t}, J=7.4 \mathrm{~Hz}, 3 \mathrm{H}) .{ }^{13} \mathrm{C}$ NMR (100 MHz, $\left.\mathrm{CDCl}_{3}\right) \delta 159.3,142.3,135.2,130.5,128.9,127.8,127.4,126.7,126.4,114.3,55.4,35.6,33.8$, 22.6, 14.2. HREIMS calcd for $\mathrm{C}_{19} \mathrm{H}_{22} \mathrm{O}\left[\mathrm{M}^{+}\right]=266.1671$, found 266.1661 .

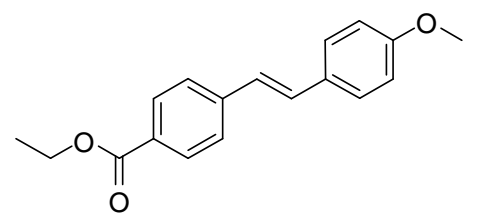

(E)-Ethyl 4-(4-methoxystyryl)benzoate. Following the general procedure using ethyl 4-iodobenzoate (84 $\mu \mathrm{L}, 0.5$ mmol) and 4-methoxystyrene $(133 \mu \mathrm{L}, 1.0 \mathrm{mmol})$, the reaction was stirred for $4 \mathrm{~h}$ at $\mathrm{rt}$. Following the standard workup, recrystallization of the crude product from warm EtOH gave $127 \mathrm{mg}(90 \%$,) of a colorless solid, $\mathrm{mp}=142$ $144{ }^{\circ} \mathrm{C}$. [Additionally $10 \mathrm{mg}(Z)$-isomer was isolated (7\%).] ${ }^{1} \mathrm{H} \mathrm{NMR}\left(400 \mathrm{MHz}, \mathrm{CDCl}_{3}\right) \delta 8.03(\mathrm{~d}, J=8.3 \mathrm{~Hz}$, 2H), $7.55(\mathrm{~d}, J=8.3 \mathrm{~Hz}, 2 \mathrm{H}), 7.49(\mathrm{~d}, J=8.6 \mathrm{~Hz}, 2 \mathrm{H}), 7.18(\mathrm{~d}, J=16.2 \mathrm{~Hz}, 1 \mathrm{H}), 7.02(\mathrm{~d}, J=16.2 \mathrm{~Hz}, 1 \mathrm{H}), 6.93(\mathrm{~d}$, 
$J=8.7 \mathrm{~Hz}, 2 \mathrm{H}), 4.39(\mathrm{q}, J=7.1 \mathrm{~Hz}, 2 \mathrm{H}), 3.85(\mathrm{~s}, 3 \mathrm{H}), 1.41(\mathrm{t}, J=7.1 \mathrm{~Hz}, 3 \mathrm{H}) .{ }^{13} \mathrm{C} \mathrm{NMR}\left(100 \mathrm{MHz}, \mathrm{CDCl}_{3}\right) \delta$ $166.5,159.8,142.2,130.7,130.0,129.6,128.9,128.2,126.1,125.5,114.3,61.0,55.4,14.5$. HREIMS calcd for $\mathrm{C}_{18} \mathrm{H}_{18} \mathrm{O}_{3}\left[\mathrm{M}^{+}\right]=282.1256$, found 282.1266 .

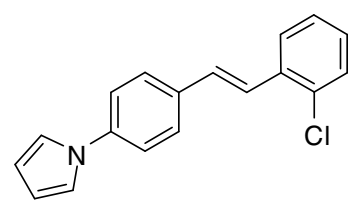

(E)-1-(4-(2-Chlorostyryl)phenyl)pyrrole. Following the general procedure using 1-(4-iodophenyl)-pyrrole (135 $\mathrm{mg}, 0.5 \mathrm{mmol})$ and 2-chlorostyrene $(128 \mu \mathrm{L}, 1.0 \mathrm{mmol})$, the reaction was stirred for $18 \mathrm{~h}$ at $\mathrm{rt}$. Following the standard workup, recrystallization of the crude product from warm EtOH gave $117 \mathrm{mg}(84 \%$,) of a light brown solid, $\mathrm{mp}=130-132{ }^{\circ} \mathrm{C}$. [Additionally $15 \mathrm{mg}(Z)$-isomer was isolated (11\%).] ${ }^{1} \mathrm{H}$ NMR $\left(400 \mathrm{MHz}, \mathrm{CDCl}_{3}\right) \delta 7.71(\mathrm{~d}, J=$ $7.8 \mathrm{~Hz}, 1 \mathrm{H}), 7.62(\mathrm{~d}, J=8.6 \mathrm{~Hz}, 2 \mathrm{H}), 7.51(\mathrm{~d}, J=16.3 \mathrm{~Hz}, 1 \mathrm{H}), 7.42(\mathrm{~m}, 3 \mathrm{H}), 7.29(\mathrm{~m}, J=6.7 \mathrm{~Hz}, 1 \mathrm{H}), 7.22(\mathrm{~m}, J=$ 7.4, $1.4 \mathrm{~Hz}, 1 \mathrm{H}), 7.14(\mathrm{~m}, J=2.0 \mathrm{~Hz}, 2 \mathrm{H}), 7.09$ (d, $J=16.3 \mathrm{~Hz}, 1 \mathrm{H}), 6.38(\mathrm{~m}, J=2.0 \mathrm{~Hz}, 2 \mathrm{H}) .{ }^{13} \mathrm{C} \mathrm{NMR}(100 \mathrm{MHz}$, $\left.\mathrm{CDCl}_{3}\right) \delta 140.4,135.5,134.6,133.6,130.3,130.1,128.8,128.2,127.1,126.6,124.8,120.6,119.3,110.8$. HREIMS calcd for $\mathrm{C}_{18} \mathrm{H}_{14} \mathrm{ClN}\left[\mathrm{M}^{+}\right]=279.0815$, found 279.0819 .



(E)-1-(2,4-Dimethylstyryl)-2-methoxynaphthalene. Following the general procedure using 1-iodo-2methoxynaphthalene (142 mg, $0.5 \mathrm{mmol})$ and 2,4-dimethylstyrene $(132 \mu \mathrm{L}, 1.0 \mathrm{mmol})$, the reaction was stirred for $24 \mathrm{~h}$ at rt. Following the standard workup, chromatography of the crude material on silica gel (1:20, EtOAc/hexanes) gave $129 \mathrm{mg}\left(89 \%\right.$,) of a tan semi-solid. [Additionally $8 \mathrm{mg}(Z)$-isomer was isolated (6\%).] ${ }^{1} \mathrm{H}$ NMR $\left(400 \mathrm{MHz}, \mathrm{CDCl}_{3}\right) \delta 8.30(\mathrm{~d}, J=8.7 \mathrm{~Hz}, 1 \mathrm{H}), 7.81(\mathrm{t}, J=7.8 \mathrm{~Hz}, 2 \mathrm{H}), 7.68(\mathrm{~d}, J=7.8 \mathrm{~Hz}, 1 \mathrm{H}), 7.47(\mathrm{t}, J=$ $7.2 \mathrm{~Hz}, 1 \mathrm{H}), 7.40-7.30(\mathrm{~m}, 4 \mathrm{H}), 7.10(\mathrm{~d}, J=8.2 \mathrm{~Hz}, 1 \mathrm{H}), 7.05(\mathrm{~s}, 1 \mathrm{H}), 3.98(\mathrm{~s}, 3 \mathrm{H}), 2.40(\mathrm{~s}, 3 \mathrm{H}), 2.37(\mathrm{~s}, 3 \mathrm{H}) .{ }^{13} \mathrm{C}$ NMR (100 MHz, $\left.\mathrm{CDCl}_{3}\right) \delta 154.8,137.4,135.7,134.6,133.0,132.8,131.3,129.5,128.8,128.5,127.1,126.6,125.5$, 124.6, 123.7, 122.5, 121.3, 113.5, 56.7, 21.3, 20.1. HREIMS calcd for $\mathrm{C}_{21} \mathrm{H}_{20} \mathrm{O}\left[\mathrm{M}^{+}\right]=288.1514$, found 288.1510.

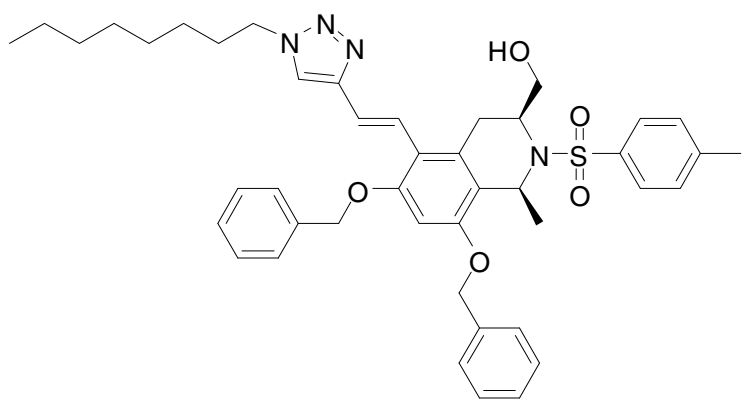

((1S,3S)-6,8-Bis(benzyloxy)-1-methyl-5-((E)-2-(1-octyl-1,2,3-triazol-4-yl)vinyl)-2-tosyl-1,2,3,4-tetra-

hydroisoquinolin-3-yl)methanol. Following the general procedure using (dtbpf) $\mathrm{PdCl}_{2}$ (2.6 mg, $0.004 \mathrm{mmol}$ ), aryl iodide 11 (134 mg, $0.20 \mathrm{mmol})$, 1-octyl-4-vinyl-1,2,3-triazole (12; $83 \mathrm{mg}, 0.40 \mathrm{mmol})$, triethylamine (84 $\mu \mathrm{L}, 0.6$ 
mmol), and $15 \mathrm{wt} \% \mathrm{PTS} / \mathrm{H}_{2} \mathrm{O}(400 \mu \mathrm{L})$ the reaction was stirred for $8 \mathrm{~h}$ at $50{ }^{\circ} \mathrm{C}$. Following the standard workup, chromatography of the crude material on silica gel (1:1, EtOAc/hexanes) gave $132 \mathrm{mg}(92 \%$,) of a light brown oil. [Additionally $5 \mathrm{mg}(Z)$-isomer was isolated (3\%).] ${ }^{1} \mathrm{H}$ NMR $\left(400 \mathrm{MHz},\left(\mathrm{CD}_{3}\right)_{2} \mathrm{CO}\right) \delta 7.96(\mathrm{~s}, 1 \mathrm{H}), 7.54(\mathrm{~d}, J=7.2$ $\mathrm{Hz}, 2 \mathrm{H}), 7.46(\mathrm{~m}, 6 \mathrm{H}), 7.41-7.29(\mathrm{~m}, 4 \mathrm{H}), 7.08$ (d, J = 8.0 Hz, 2H), 6.88 (d, J = 16.5 Hz, 1H), 6.64 (s, $1 \mathrm{H}), 5.44(\mathrm{q}$, $J=7.0 \mathrm{~Hz}, 1 \mathrm{H}), 5.18-5.07(\mathrm{~m}, 4 \mathrm{H}), 4.38(\mathrm{t}, J=7.0 \mathrm{~Hz}, 1 \mathrm{H}), 4.14(\mathrm{t}, J=6.7 \mathrm{~Hz}, 1 \mathrm{H}), 3.84(\mathrm{~m}, 1 \mathrm{H}), 3.82(\mathrm{~m}, 2 \mathrm{H})$, $3.36(\mathrm{dd}, J=16.1,6.5 \mathrm{~Hz}, 1 \mathrm{H}), 2.88(\mathrm{~m}, 2 \mathrm{H}), 2.26(\mathrm{~s}, 3 \mathrm{H}), 1.89(\mathrm{~m}, 2 \mathrm{H}), 1.45(\mathrm{~d}, J=7.0 \mathrm{~Hz}, 3 \mathrm{H}), 1.38-1.24(\mathrm{~m}$, $10 \mathrm{H}), 0.87(\mathrm{t}, J=7.1 \mathrm{~Hz}, 3 \mathrm{H}) .{ }^{13} \mathrm{C} \mathrm{NMR}\left(100 \mathrm{MHz}, \mathrm{CDCl}_{3}\right) \delta 156.8,153.4,146.8,143.0,137.1,136.7,135.6$, 133.4, 129.2, 129.0, 128.8, 128.3, 128.1, 127.4, 127.2, 127.1, 123.6, 121.7, 120.2, 117.9, 96.3, 71.0, 70.1, 67.4, 56.3, 50.5, 47.9, 31.9, 30.5, 29.2, 29.1, 26.6, 26.5, 23.0, 22.8, 21.6, 14.2. HREIMS calcd for $\mathrm{C}_{44} \mathrm{H}_{52} \mathrm{~N}_{4} \mathrm{O}_{5} \mathrm{~S}[\mathrm{M}+\mathrm{Na}]=$ 771.3556, found 771.3515 .

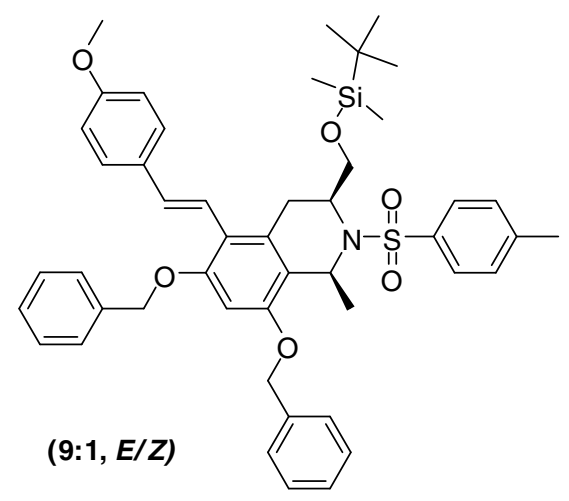

Table 3, entry 7. Following the general procedure using (dtbpf) $\mathrm{PdCl}_{2}(1.0 \mathrm{mg}, 0.0015$ mmol), aryl iodide 13 (50 $\mathrm{mg}, 0.063 \mathrm{mmol})$, 4-methoxystyrene $(20 \mu \mathrm{L}, 0.127 \mathrm{mmol})$, triethylamine $(27 \mu \mathrm{L}, 0.189 \mathrm{mmol})$, and 15 wt.\% PTS/ $\mathrm{H}_{2} \mathrm{O}(200 \mu \mathrm{L})$ the reaction was stirred for $8 \mathrm{~h}$ at rt. Following the standard workup, chromatography of the crude material on silica gel (1:10, EtOAc/hexanes) gave $45 \mathrm{mg}(90 \%$,$) of a colorless semi-solid (9:1 isomeric$ mixture, inseparable). ${ }^{1} \mathrm{H}$ NMR $\left(400 \mathrm{MHz}, \mathrm{CDCl}_{3}\right.$ ) 'see below'. HREIMS calcd for $\mathrm{C}_{47} \mathrm{H}_{55} \mathrm{NO}_{6} \mathrm{SSi}[\mathrm{M}+\mathrm{Na}]=$ 812.3417, found 812.3431.

\section{References}

[1] (a) Tang, Y.; Yu, Y.; Xia, W.; Song, Y. Huang, Z.; J. Org. Chem. 2002, 67, 3096-3103. (b) List, B.; Doehring, A.; Fonseca, M.; Job, A.; Torres, R. Tetrahedron, 2006, 62, 476-482. (c) Herrmann, W.; Bohm, V. J. Organomet. Chem. 1999, 572, 141-145. (d) Vazquez, E.; Caron, S.; Stevens, R.; Nakao, K.; Koike, H.; Yoshinori. M.; J. Org. Chem. 2003, 68, 4104-4107. (e) Shi, M.; Xu, B. J. Org. Chem. 2002, 67, 294-297. 








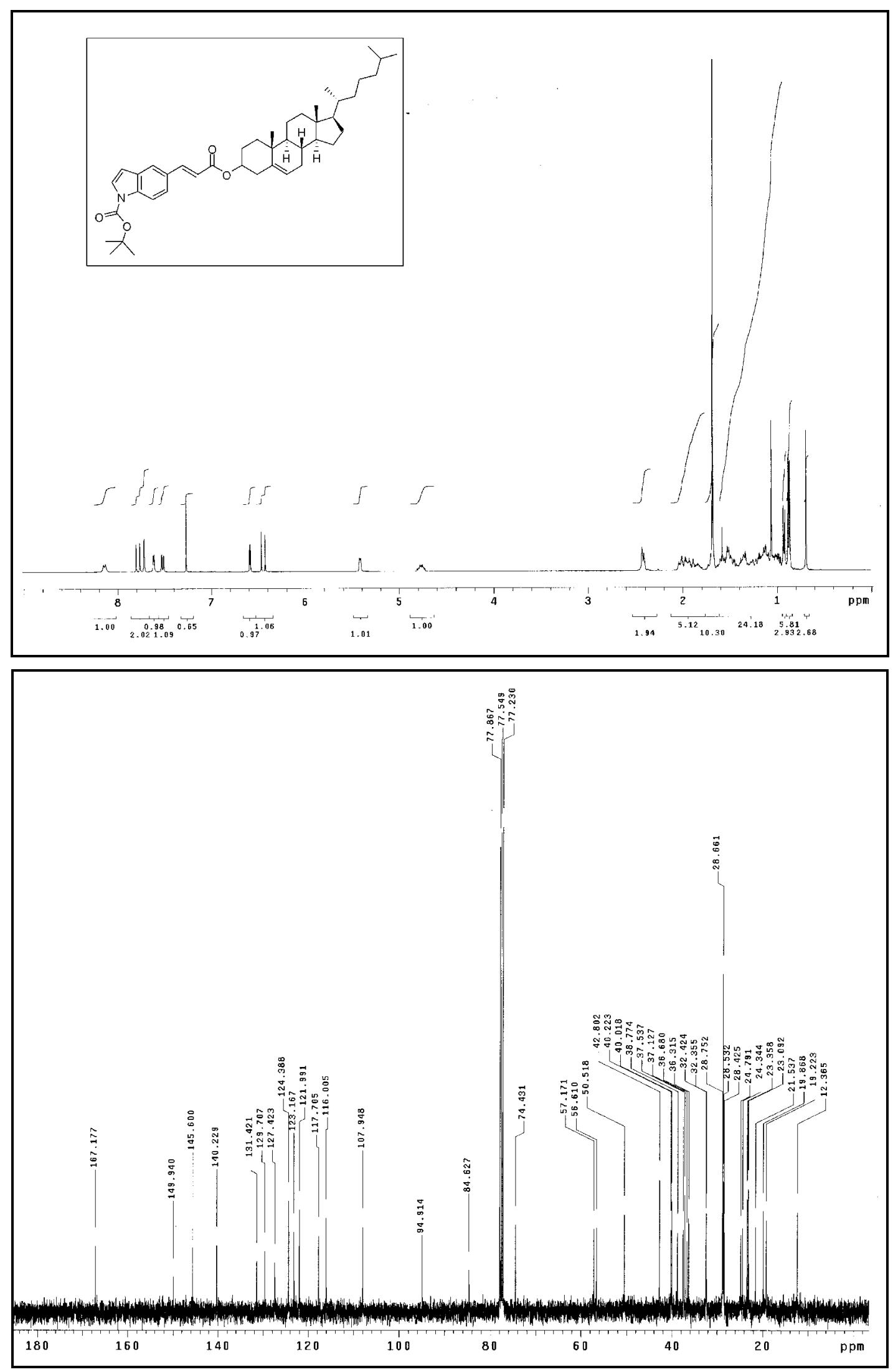


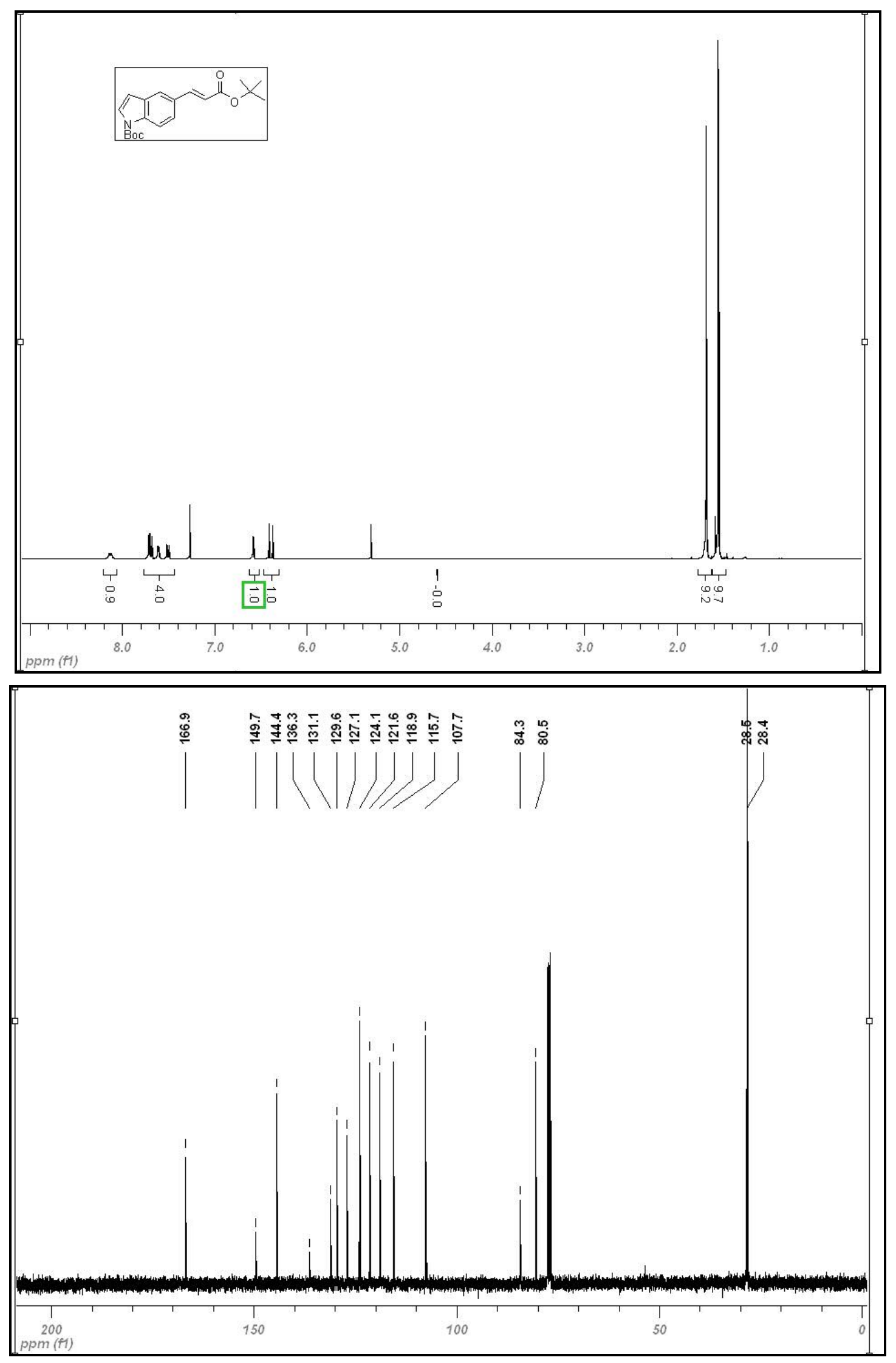




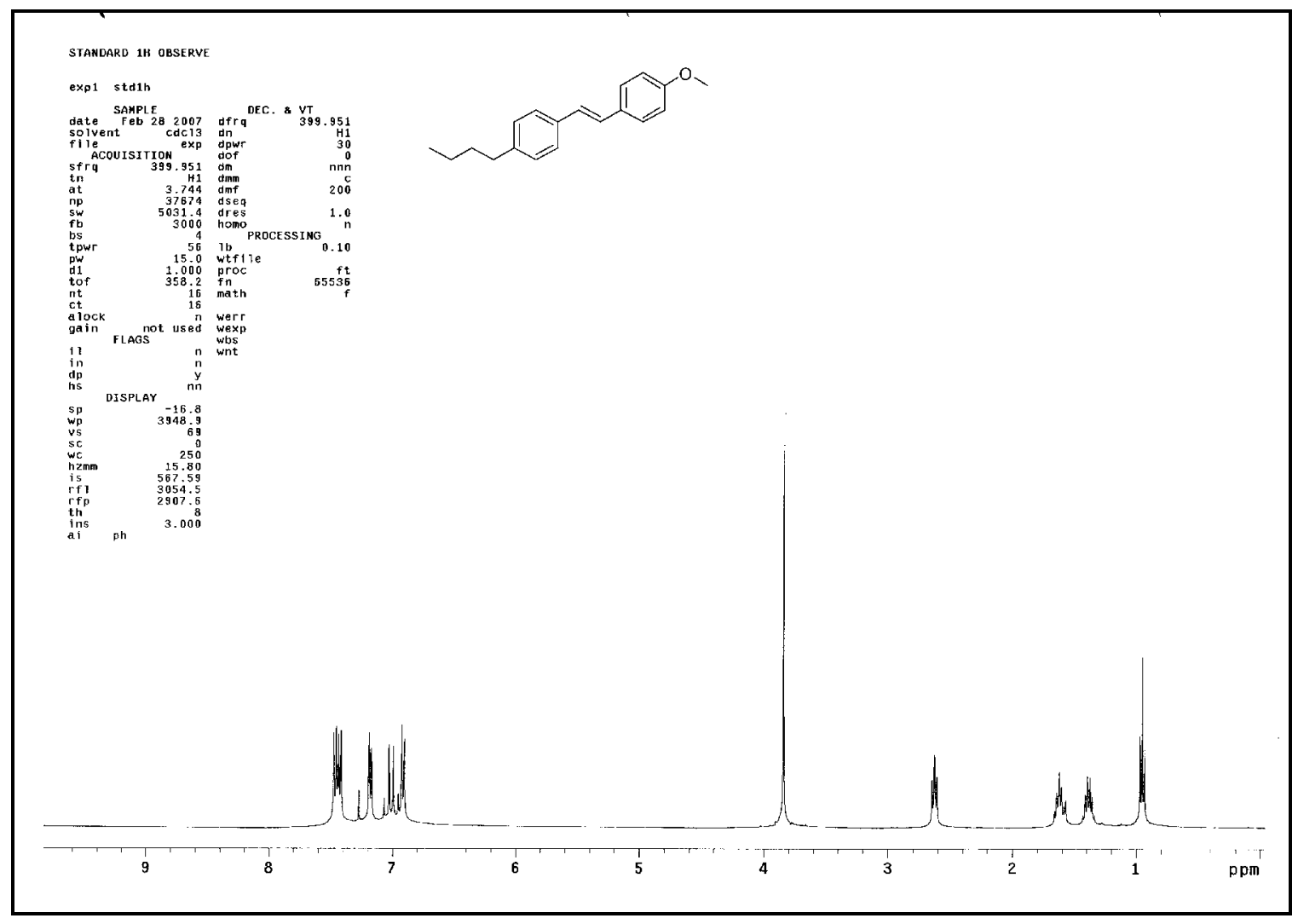

Standard c13 run using anp probe

Pulse Sequence: s2pul

Solvent: cdelo
Ambient temperature

Relax de lay $9.000 \mathrm{sec}$
Pulse 69.2 derees

Acq in $25000.00 \mathrm{~Hz}$

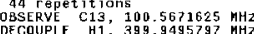

Dewer 42 dB
cont inuously on

WALTZ-16 MOdu IT
DATA PROCESSING

DATA PRDCESSING $0.5 \mathrm{~Hz}$

FT $s 12$ e 65536
Total time $9 \mathrm{~min}, 55 \mathrm{sec}$

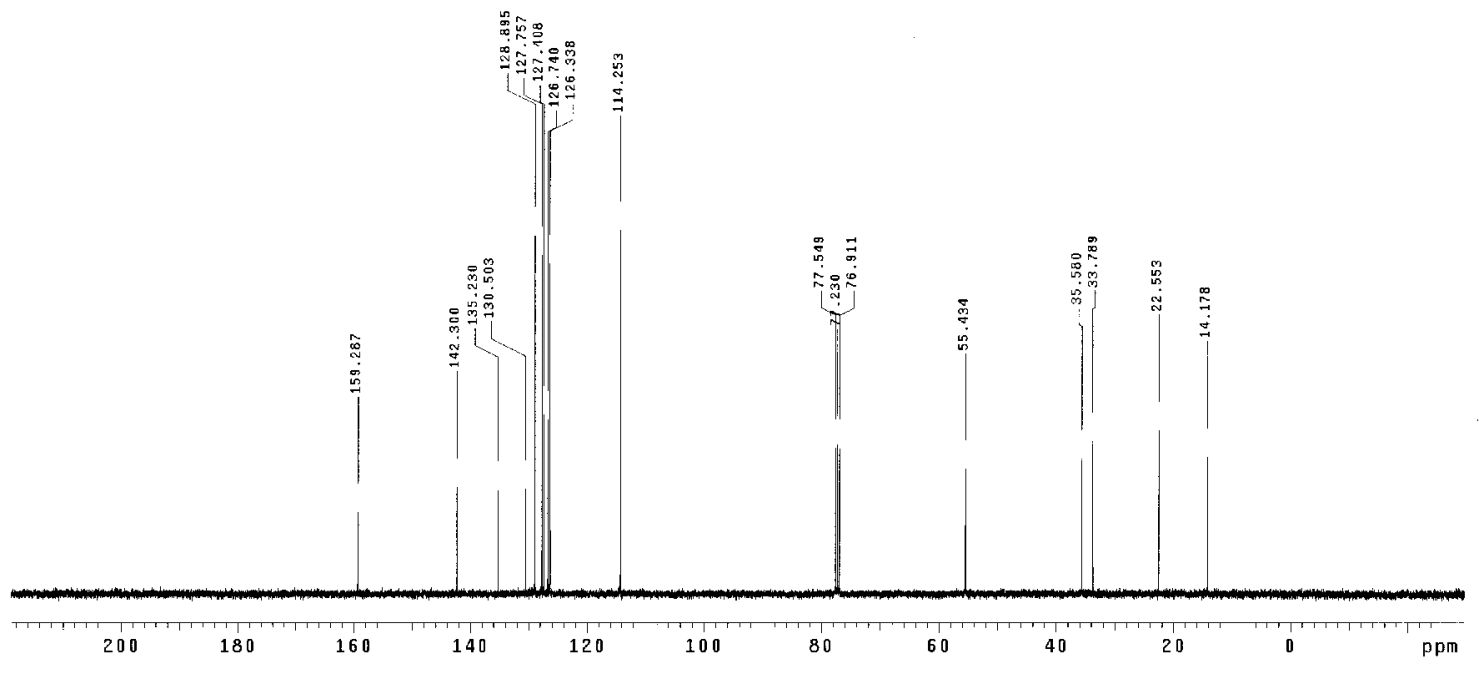




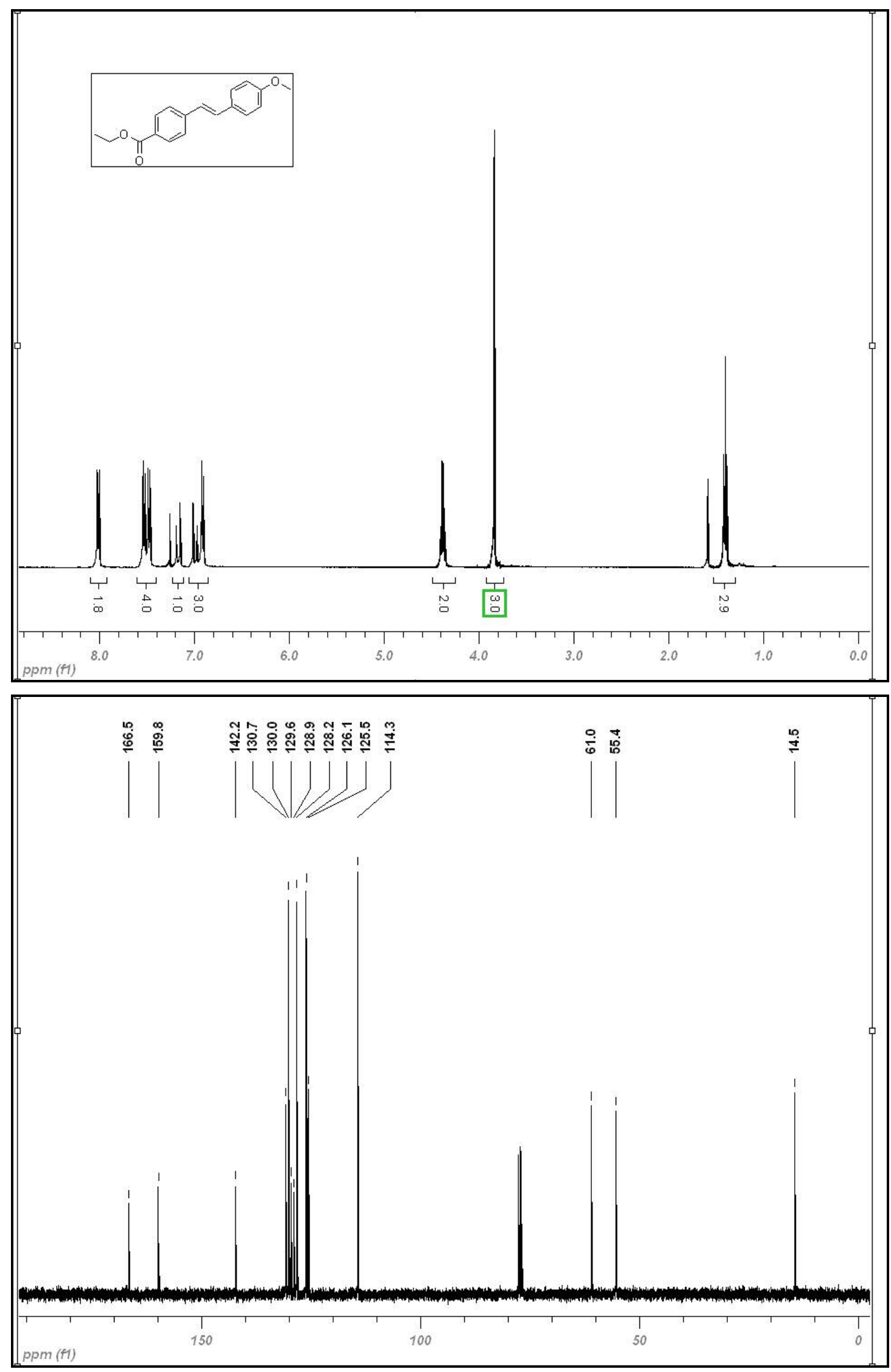



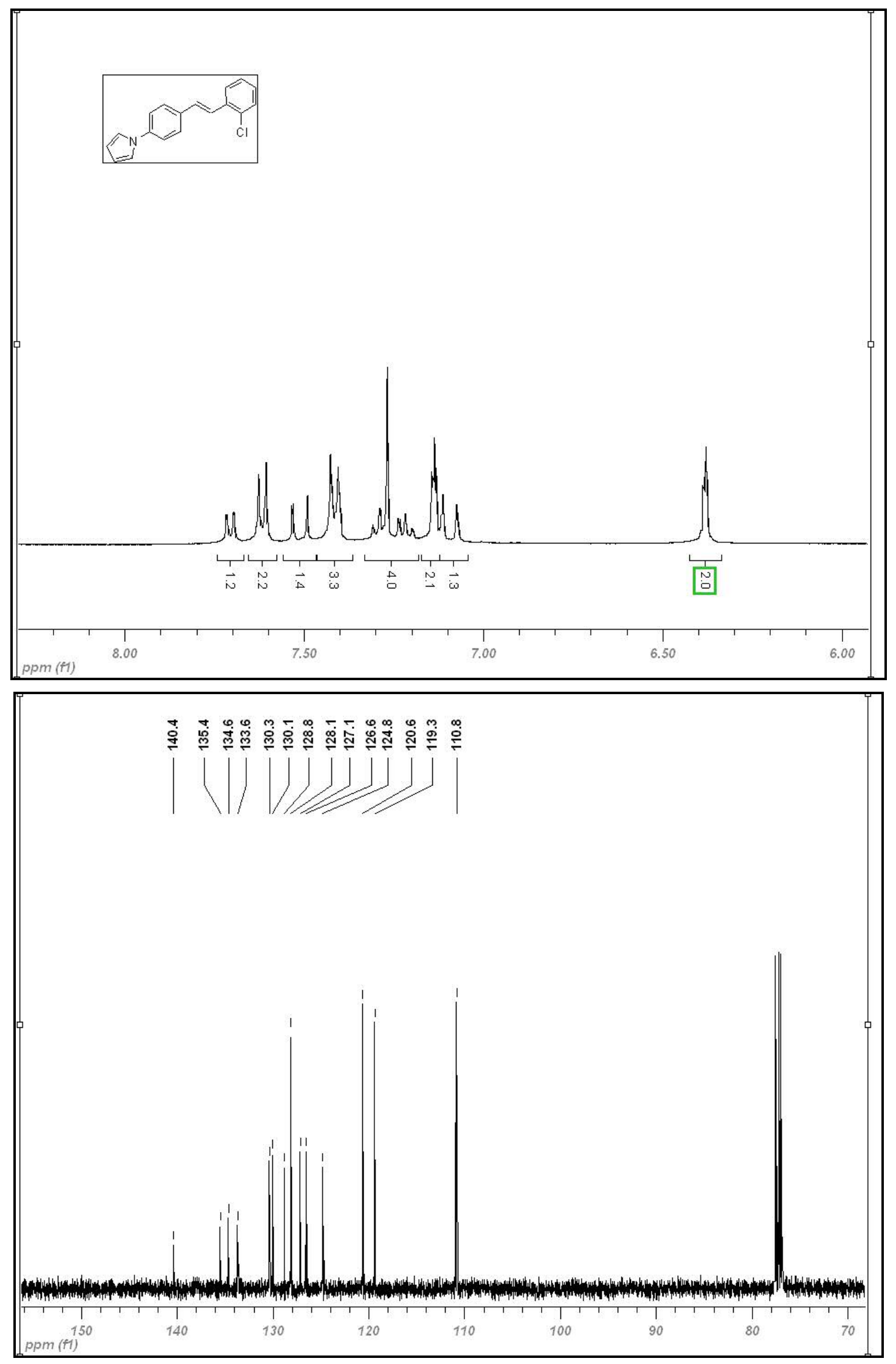

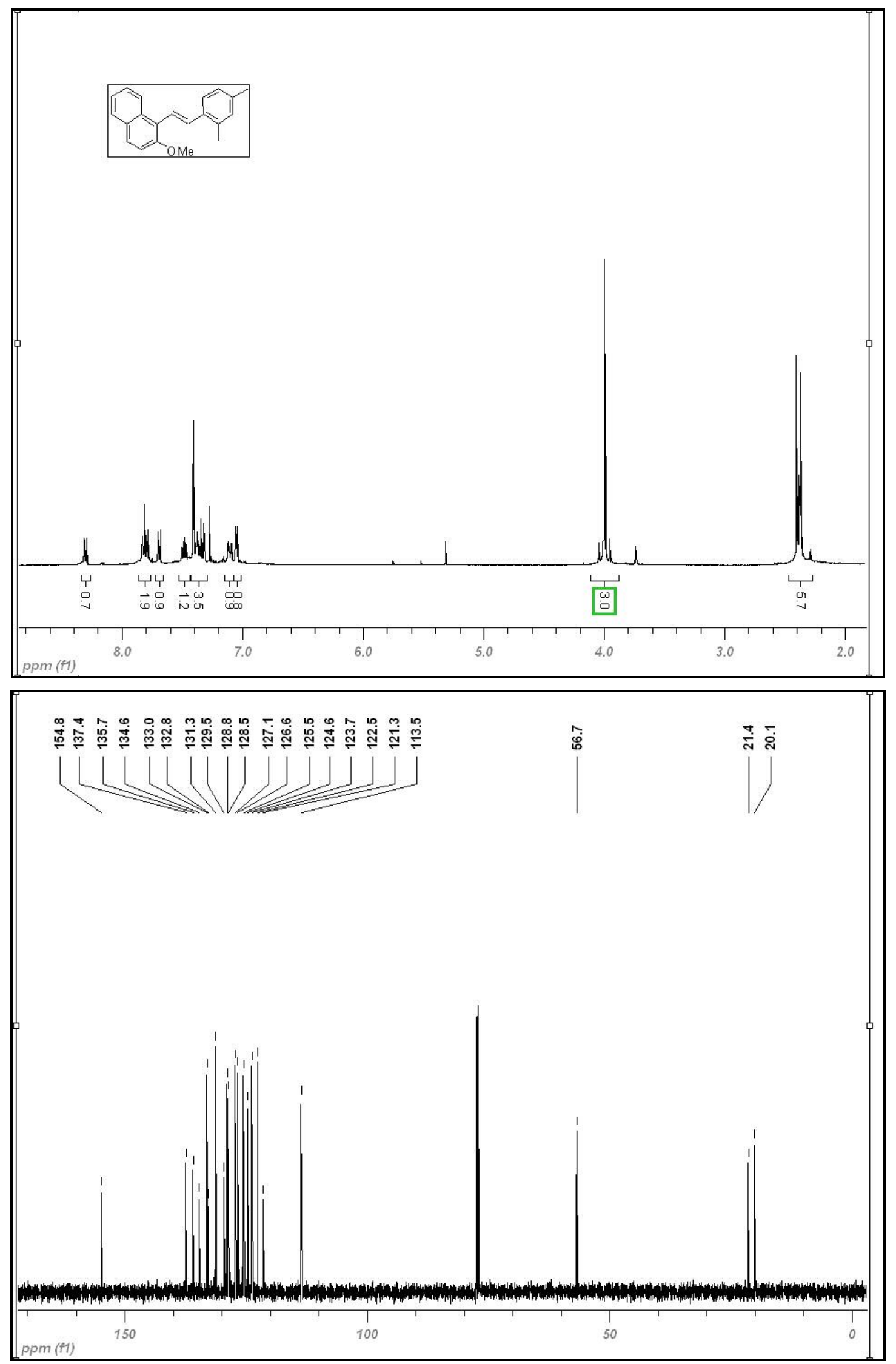

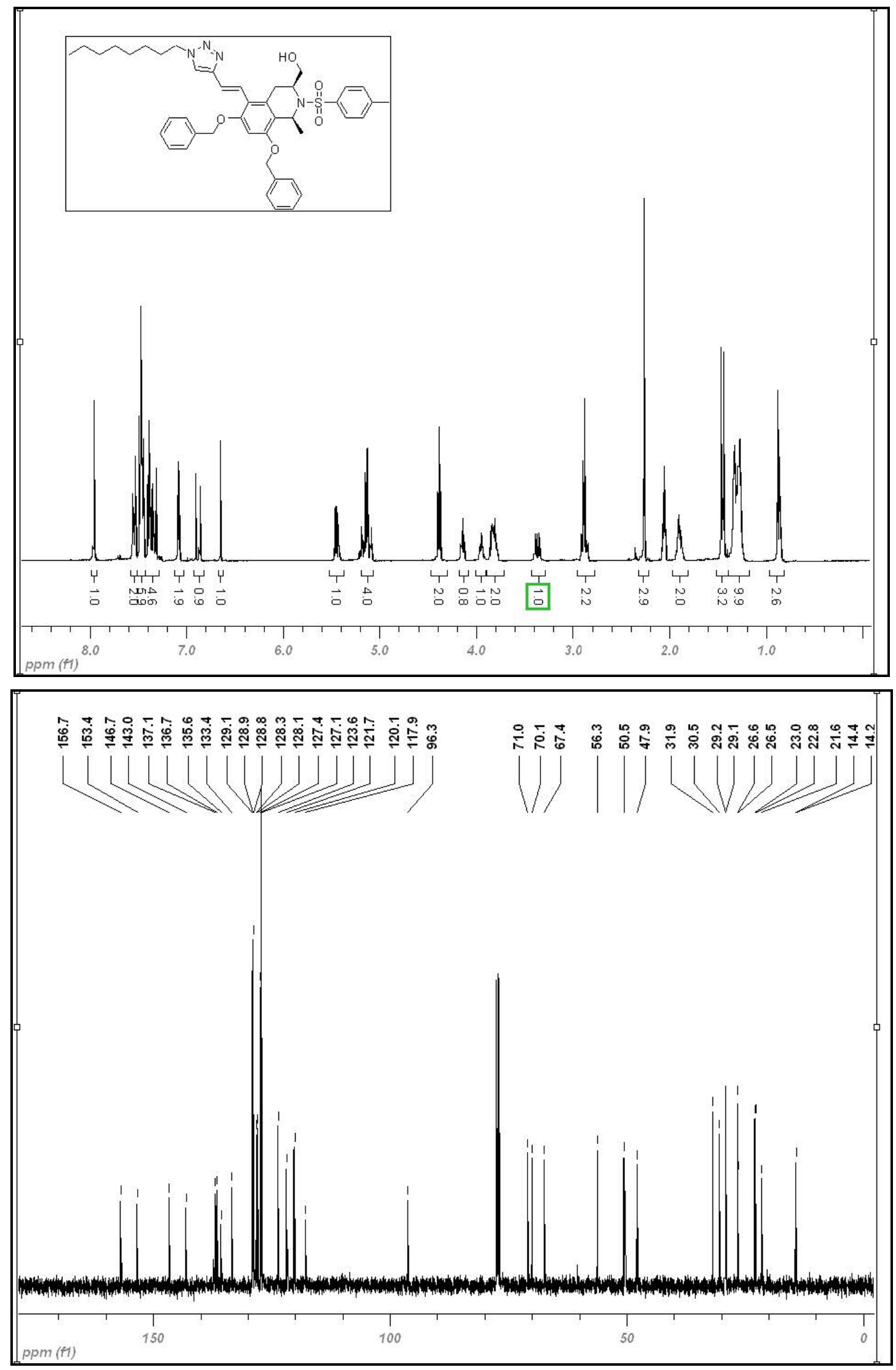


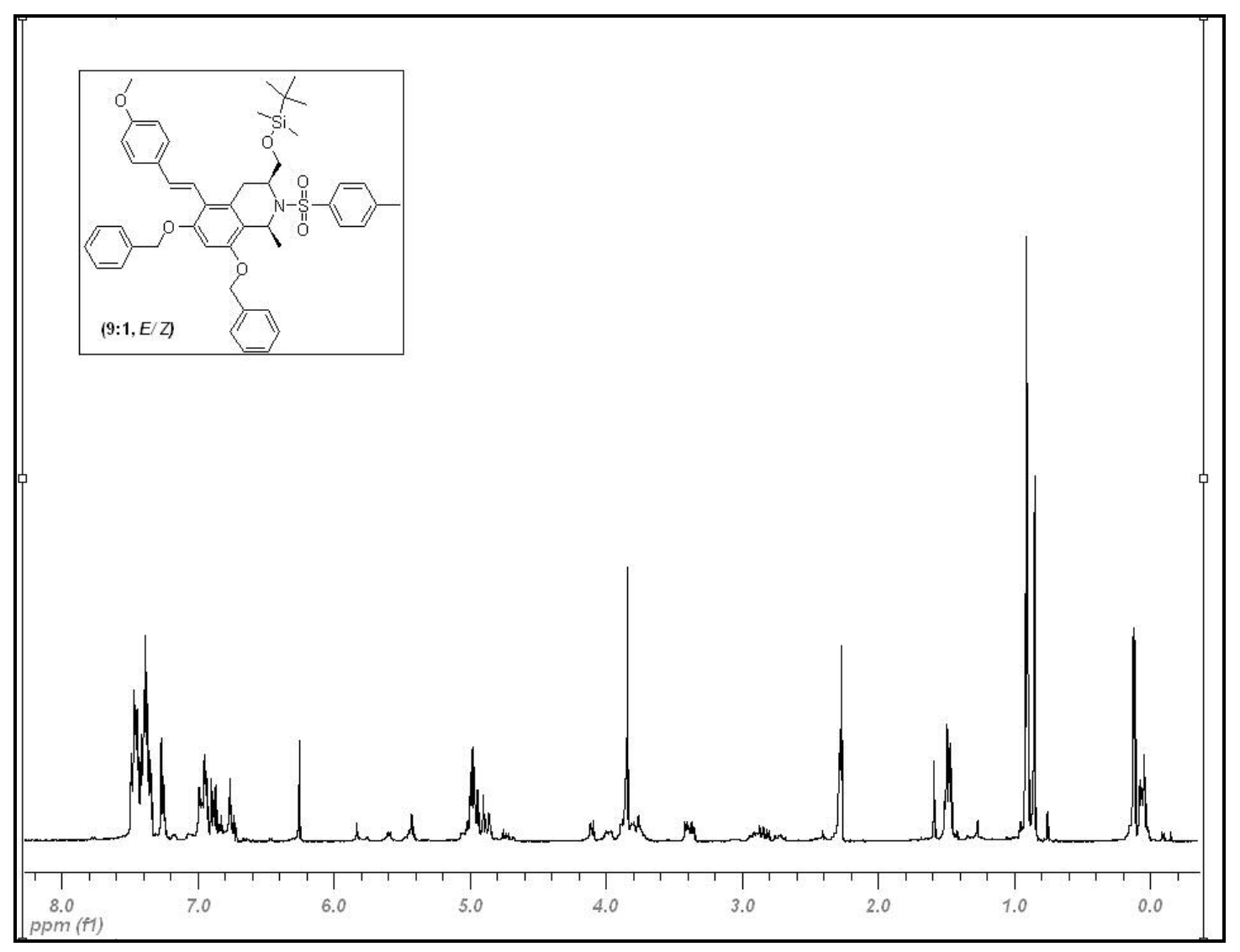

